\title{
Zeolite-Catalyzed Hydrocarbon Formation from Methanol: Density Functional Simulations
}

\author{
Niranjan Govind**, Jan Andzelm, Kurt Reindel, George Fitzgerald \\ Accelrys Inc., 9685 Scranton Road, San Diego, California 92121, USA
}

**Author to whom correspondence should be addressed. Email: nxg@accelrys.com

Received: 16 October 2001 / Accepted: 31 January 2002 / Published: 25 April 2002

\begin{abstract}
We report detailed density functional theory (DFT) calculations of important mechanisms in the methanol to gasoline (MTG) process in a zeolite catalyst. Various reaction paths and energy barriers involving $\mathrm{C}-\mathrm{O}$ bond cleavage and the first $\mathrm{C}-\mathrm{C}$ bond formation are investigated in detail using all-electron periodic supercell calculations and recently developed geometry optimization and transition state search algorithms. We have further investigated the formation of ethanol and have identified a different mechanism than previously reported [1], a reaction where water does not play any visible role. Contrary to recent cluster calculations, we were not able to find a stable surface ylide structure. However, a stable ylide structure built into the zeolite framework was found to be possible, albeit a very high reaction barrier.
\end{abstract}

Keywords: Density functional theory, MTG, methanol, hydrocarbons, gasoline, zeolite, catalyst, supercell, geometry optimization, transition state.

\section{Introduction}

Zeolites are water-containing crystalline, porous aluminosilicates composed of $\mathrm{SiO}_{4}$ and $\mathrm{AlO}_{4}{ }^{-}$ edge-sharing tetrahedra interlinked through common oxygen atoms giving rise to three-dimensional networks of channels, cages and rings. These structural attributes account for the different physical properties of individual zeolites. Since silicon is balanced by four surrounding oxygen atoms, in a pure silicate, a zeolite is charge neutral. However, when one substitutes silicon atoms by aluminum the charge balance of the network is upset creating strong Brønsted acid sites. Zeolites are thus very good solid-acid catalysts. Two important industrially relevant processes involving zeolites include the 
conversion of methanol to gasoline (MTG) and methanol to olefins (MTO) which were developed by Mobil in the 1970s. A thorough understanding of the various reactions involved in the MTG process has been the subject of industrial and academic research for many years. In particular, researchers have been interested in the details in the mechanism of the $\mathrm{C}-\mathrm{O}$ bond cleavage in methanol and the formation of the first $\mathrm{C}-\mathrm{C}$ bond. A number of reaction mechanisms have been proposed with supporting experimental evidence in some cases [2-4]. The commonly accepted mechanism involves the initial physisorption of methanol at a Brønsted acid site of the Al-substituted zeolite [5]. As the concentration of methanol increases, clusters of hydrogen-bonded methanol molecules are formed in the zeolite cage [6,7]. At that stage di-methyl-ether DME can be formed [8], as confirmed by experiment [2]. It is, however, unclear whether the formation of DME is a necessary intermediate for the first $\mathrm{C}-\mathrm{C}$ bond formation [9]. As for the $\mathrm{C}-\mathrm{O}$ bond cleavage in methanol, it can occur through the formation of surface methoxyl species [6,10-12] This reactive species then serves as a starting point for the formation of the initial C-C bond leading to the formation of ethanol or ethyl-methyl-ether [13]. These intermediates can be dehydrated easily, thereby producing ethylene, which can undergo further reactions leading to the formation of higher olefins, alkanes aromatics and naphtenes that make up standard commercial gasoline [13]. Recently, yet another mechanism for the C-C bond formation has been proposed: this involves the formation of a surface ylide neighboring a Brønsted acid site $[9,13]$. In principle, the surface ylide species can form from the methoxyl species via proton (or hydride) transfer to a neighboring bridging oxygen. However, it is also possible that the ylide carbon atom is incorporated into the zeolite framework leading to a significant reorganization of a large number of atoms around the Brønsted acid site.

First principles studies involving zeolites have commonly employed cluster models to represent the neighborhood of the Brønsted acid site [4,6 and 9-13]. There have only been a few calculations that have utilized periodic boundary conditions to represent the realistic, crystalline environment for the MTG reaction [1,5,7,8]. Plane-wave DFT calculations, for instance, have been used to study the physisorption and clustering of methanol molecules leading to the formation of DME $[7,8]$. In our first paper [1] we reported the first periodic calculations investigating the formation of a surface methoxyl species and the formation of a ethanol within a zeolite cage. The possibility of surface ylide formation as well as ylide incorporation into the framework close to a Brønsted acid site was also studied [1]. In this paper we discuss in more detail the above mentioned processes. A new reaction path for ethanol formation is described as well.

\section{Computational Details}

All-electron periodic DFT calculations reported here were performed using the $\mathrm{DMol}^{3}$ program $[14,15]$. The electronic wavefunctions are expanded in atom-centered basis functions defined on a dense numerical grid. We used the double-numeric-polarized (DNP) [14] basis set, and an integration grid that amounted to approximately 5500 grid points per atom. The DNP, all-electron basis set is 
composed of two numerical functions per valence orbital, supplemented with a polarization function. Each basis function was restricted to a cutoff radius of $R_{\text {cut }}=4.0 \AA$, thereby allowing for efficient calculations without a significant loss of accuracy. The electron density was approximated using a multipolar expansion up to hexadecapole and the exchange-correlation was represented using the gradient-corrected PBE functional [20]. Brillouin-zone integrations were performed using a 2 x 2 × 2 Monkhorst-Pack (MP) grid [21].

All geometry optimizations were performed using a recently developed scheme based on delocalized internal coordinates generalized to periodic boundary conditions [18]. It has been shown [18] that for systems such as zeolites this algorithm can be several times more efficient than ones based on Cartesian coordinates. For example, full geometry optimization of the Chabazite zeolite structure based on Cartesian coordinates is achieved in about 96 steps whereas it takes only 21 steps with the optimizer based on delocalized internal coordinates clearly demonstrating the speedup. Methanol and water molecules in zeolite pores present an additional challenge for the optimization algorithm, since these molecules are typically not connected to the zeolite framework. The DMol ${ }^{3}$ geometry optimizer allows for a treatment of such disconnected fragments without the need for any artificial connecting bonds. Several reaction paths investigated here required an approximate scan of the potential energy surface. This was accomplished using internal constraints that were imposed on connected or disconnected fragments. However, precise determination of the transition states is a formidable challenge as it involves many a concerted reaction within the zeolite cage. The traditional method of calculating a Hessian and following the reaction mode can be very expensive for any technologically important zeolite. Moreover, since these methods are based on quadratically convergent schemes, the success of such calculations is not guaranteed unless the guess structure is already close to the transition state. In this paper we employed a recently developed Cartesian based algorithm [19] that blends a generalization of the synchronous transit (ST) method with a conjugate gradient $(\mathrm{CG})$ refinement technique. It only requires the reactant and product structures and the transition state is located iteratively via a series of ST/CG steps. This scheme has been found to be robust, efficient and accurate and can be applied to both molecular and periodic systems in a seamless fashion.

\section{Results}

\section{Hydrogen bonding}

The MTG reaction mechanism is determined to a large extent by the network hydrogen bonds connecting methanol, water molecules and the $\mathrm{H}$-atom at the Brønsted acid site. An accurate treatment of hydrogen bonds is, therefore, necessary for this study. To test the accuracy of $\mathrm{DMol}^{3}$ in describing H-bond strengths, the interaction energies between two methanol molecules, and between two water molecules were computed using the $\mathrm{PBE} / \mathrm{DNP} / \mathrm{R}_{\mathrm{cut}}=4.0 \AA$ settings described in the previous section 
(Figure1). The calculated values of 5.8 and $5.7 \mathrm{kcal} / \mathrm{mol}$ for methanol and water dimers compare well with experimentally estimated ranges of 4.6-5.9 and 5.0-5.4 kcal/mol [22], respectively. This good agreement is due to the fact that the interaction is dominated by the electrostatics, and DFT predicts dipole moments for both methanol and water with reasonable accuracy [23]. Dispersion forces that are absent in DFT [24] and contribute perhaps as much as $25 \%$ to the total interaction energy of water dimer [22] are apparently compensated by other terms in the interaction energy. Since DMol ${ }^{3}$ utilizes atom centered basis functions, the convergence of interaction energies with respect to the size of the basis set was also carefully checked. Traditional ab initio calculations use the basis set superposition error (BSSE) approach [22] to estimate the errors associated with the use of incomplete basis sets. $\mathrm{DMol}^{3}$ uses numerical functions that are far more complete than traditional Gaussian functions, and therefore we expect BSSE contribution to be small. We verified this by performing calculations on a water dimer using a much larger basis set (five numerical functions per every valence orbital, supplemented with three sets of polarization functions, diffuse functions and $R_{\text {cut }}=8.0 \AA$ ). These calculations affect the interaction energy by less than $0.5 \mathrm{kcal} / \mathrm{mol}$. Therefore, indirectly, we demonstrate that any BSSE contributions or further basis set extensions have a small effect of a fraction of a $\mathrm{kcal} / \mathrm{mol}$ on the interaction energy. This convergence was also verified using the pseudopotential based plane wave program CASTEP [16,17] where the size of the basis can be systematically increased via the energy cutoff. Extremely well converged calculations $\left(\mathrm{E}_{\mathrm{cut}}=400 \mathrm{eV}\right)$ yielded 6.2 and $5.9 \mathrm{kcal} / \mathrm{mol}$ with the PW91 functional for the methanol and water dimers compared with 6.2 and $6.1 \mathrm{kcal} / \mathrm{mol}$ obtained using $\mathrm{DMol}^{3}$ with the same functional. Similar converged results were obtained using the PBE functional: 5.5 and $5.4 \mathrm{kcal} / \mathrm{mol}$ (CASTEP), 5.8 and $5.7 \mathrm{kcal} / \mathrm{mol}$ $\left(\mathrm{DMol}^{3}\right)$. The small differences could be attributed to the pseudopotentials used in plane wave calculation. Nevertheless, these results confirm the flexibility and convergence of our basis set.

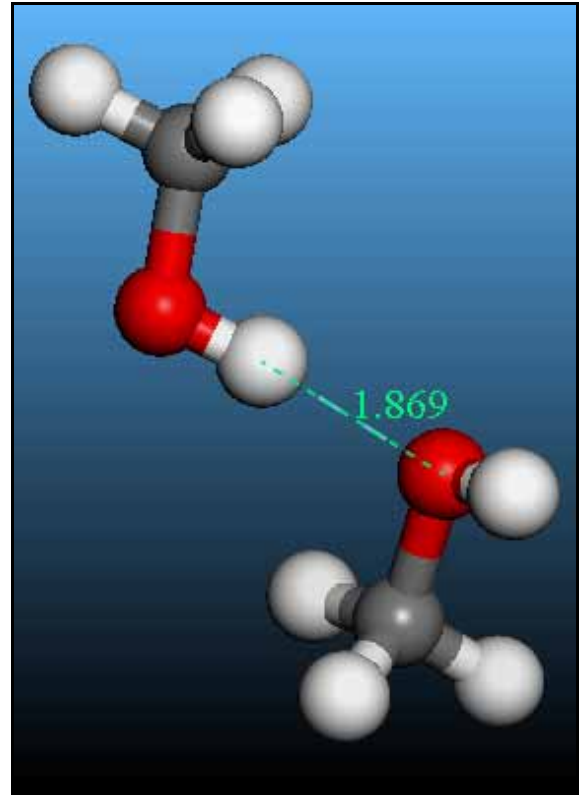

(a)

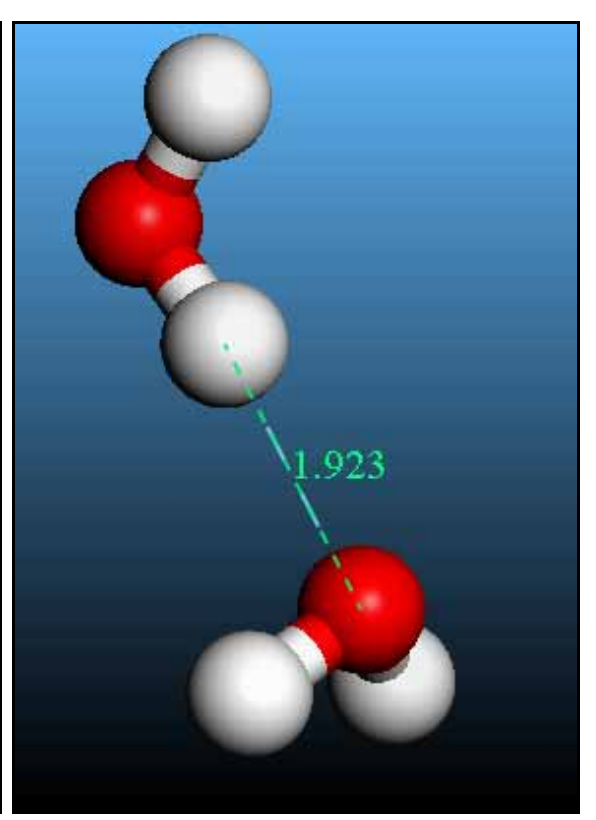

(b)

Figure 1. (a) Methanol dimer, (b) Water dimer with hydrogen bonds and distances shown. 


\section{Bridging hydroxyl groups and zeolite models}

A thorough study of all reactions involved in the MTG process in commercially relevant zeolites can become computationally intensive as they involve very large unit cells. The unit cell of the ZSM-5 catalyst, for instance, has 288 atoms. In this work we have chosen the Ferrierite (FER) structure that has eight- and ten-ring channels - somewhat similar to the ten-ring channels of the industrially important ZSM-5. The unit cell of FER contains 54 atoms, making it an excellent system for detailed electronic structure studies. Recent calculations by Haase and Sauer [5] on the physisorption of a single methanol molecule in the FER and ZSM-5 zeolites show similar complexes in both zeolites. The methanol adsorption energies in both zeolites differ by less than $4 \mathrm{kcal} / \mathrm{mol}$, which is only $15 \%$ of the adsorption energy. One, therefore, expects most results from the calculations on the FER system to remain valid for a commercial zeolite like ZSM-5.

To assess the accuracy of the DMol3 settings in a periodic geometry of a zeolite, we performed calculations on bridging hydroxyl groups at the alumino-silicate Brønsted acid site of the Faujasite (FAU) and FER zeolites. Experimental studies [25] on the FAU zeolite have revealed that only three of the four possible bridging $\mathrm{OH}$ groups are observed and their relative occupations are as follows: 3:1:1.6:0 for $\mathrm{O} 1 \mathrm{H}: \mathrm{O} 2 \mathrm{H}: \mathrm{O} 3 \mathrm{H}: \mathrm{O} 4 \mathrm{H}$ sites, respectively. Hill et al. [26] using the VWN/DNP settings found excellent correlation between the relative energies of the four bridging $\mathrm{OH}$ groups and the relative experimental occupations. In this work geometry optimization was performed on all the 145 atoms of the rhombohedral cell with fixed and optimized cell parameters. Table 1 summarizes our results as compared with the results of Ref. [26]. It is evident that the relative energies from our calculations correspond well with the experimental site occupations as well as with previous calculations and the overall picture remains the same for the fixed and optimized cases, respectively. The bond lengths and bond angles associated with bridging hydroxyl groups are within $0.06 \AA$ and $6^{\circ}$ of experimental values. The $\mathrm{O} 1 \mathrm{H}$ site, which is open to the largest channel of the zeolite, is a preferable location for the hydroxyl group in both FAU and FER zeolites. In the case of the FER zeolite, the relative energies are $0,7.5,9.5$, and $14.2 \mathrm{~kJ} / \mathrm{mol}$ for the $\mathrm{O} 1 \mathrm{H}, \mathrm{O} 2 \mathrm{H}, \mathrm{O} 3 \mathrm{H}$, and $\mathrm{O} 4 \mathrm{H}$ sites, respectively. The $\mathrm{O} 1 \mathrm{H}$, being the lowest energy site, was selected as the Brønsted acid site for our MTG study with the FER zeolite.

Table 1. Selected geometrical parameters and relative energies of the four bridging $\mathrm{OH}$ groups in $\mathrm{H}-$ faujasite (FAU). Experimental values are shown in parentheses [25, 26]

\begin{tabular}{llcccc}
\hline & & & \multicolumn{3}{c}{ Energy $(\mathrm{kJ} / \mathrm{mol})$} \\
\cline { 4 - 6 } Site & \multicolumn{1}{c}{$\mathrm{r}_{\mathrm{AlH}}(\AA)$} & $\alpha_{\mathrm{SiO}(\mathrm{H}) \mathrm{Al}}(\mathrm{deg})$ & Fixed Cell & Ref. [26] & Optimized Cell \\
\hline $\mathrm{O} 1 \mathrm{H}$ & $2.54(2.48 \pm 04)$ & $130.1(135.7)$ & 0.0 & 0.0 & 0.0 \\
$\mathrm{O} 2 \mathrm{H}$ & 2.43 & $141.1(144.6)$ & 7.8 & 9.8 & 8.0 \\
$\mathrm{O} 3 \mathrm{H}$ & $2.46(2.40 \pm 04)$ & $136.8(139.8)$ & 4.1 & 4.9 & 4.3 \\
$\mathrm{O} 4 \mathrm{H}$ & 2.44 & $135.9(141.9)$ & 9.4 & 7.9 & 9.1 \\
\hline
\end{tabular}




\section{Surface methoxyl: Formation and Reactions}

A single molecule of methanol forms multiple hydrogen bonds with the Brønsted acid site of FER zeolite. There are several adsorption sites possible that are within $1 \mathrm{kcal} / \mathrm{mol}$; Figure $2 \mathrm{a}$ displays the most stable structure with an adsorption energy of $18.5 \mathrm{kcal} / \mathrm{mol}$. This value compares well with the experimental estimates of the heat of adsorption in acidic zeolites, ranging from 15 to $27 \mathrm{kcal} / \mathrm{mol}$ $[6,27]$. No protonation of methanol by the Brønsted acid site was found, in agreement with the recent study by Haase and Sauer [5]. The calculated transition state for the methylation of a surface oxygen at the alumino-silicate Brønsted acid site of FER zeolite is presented in Figure. 2b. This is a concerted reaction involving breaking of the $\mathrm{C}-\mathrm{O}$ bond in methanol and bond formation between $\mathrm{C}$ and surface oxygen. The proton is simultaneously transferred from the Brønsted acid site to the hydroxyl group, thereby forming a water molecule. The activation barrier for this reaction was found to be $54 \mathrm{kcal} / \mathrm{mol}$ which is not surprising because this involves a strained $\mathrm{S}_{\mathrm{N}} 2$ type process as can be clearly seen from the figure (see Figure 2). Other DFT or ab initio cluster studies [10-12] have reported a barrier of 44 to $65 \mathrm{kcal} / \mathrm{mol}$.

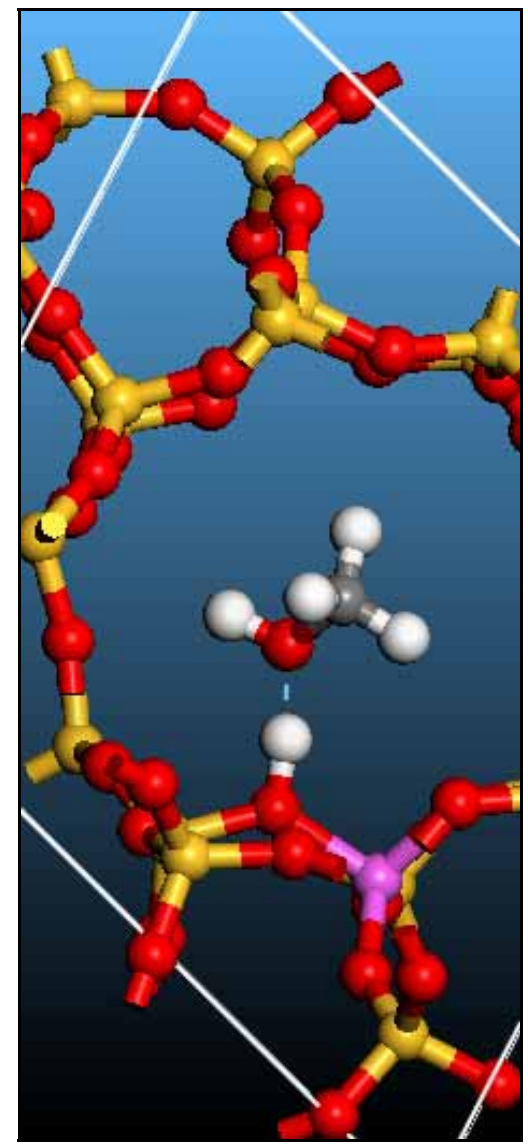

(a)

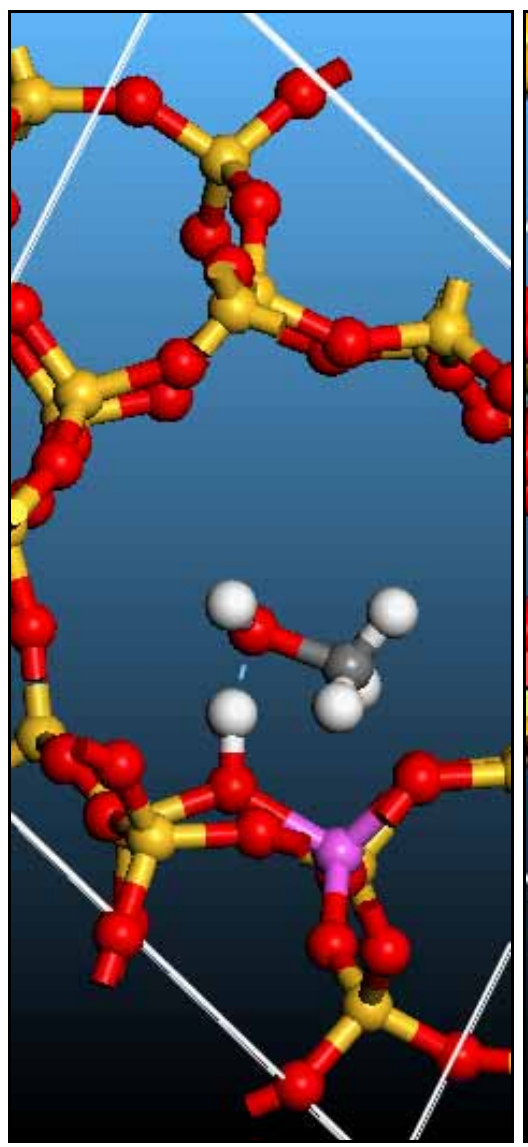

(b)

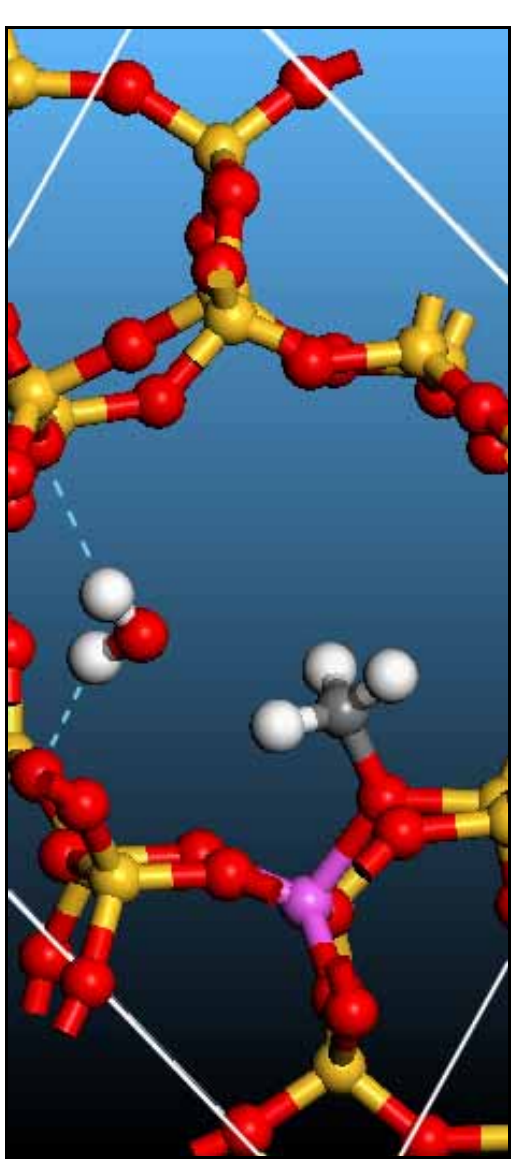

(c)

Figure 2. Surface methoxyl formation with a single methanol molecule via a strained $S_{N} 2$ type reaction. (a) Hydrogen-Methanol complex at the Brønsted acid site, (b) Transition state structure, (c) Surface methoxyl. Dotted lines represent hydrogen bonds. 
Introducing a second methanol, interestingly, lowers the above activation barrier to $44 \mathrm{kcal} / \mathrm{mol}$. This can be understood easily as this transition state now corresponds to an unstrained $\mathrm{S}_{\mathrm{N}} 2$ pathway (see Figure 3). At the transition state the surface oxygen, methyl carbon and the oxygen of the water molecule that leaves are roughly collinear (Figure $3 b$ ). The water molecule is formed as a result of proton transfer to the methanol hydroxyl group from the methoxonium ion. Our computed barrier of 44 $\mathrm{kcal} / \mathrm{mol}$ is comparable with $32-46 \mathrm{kcal} / \mathrm{mol}$ as predicted by the MP2/5-31G*//HF/3-21G studies of Sinclair and Catlow [6] on clusters of various sizes. This reaction is facilitated by hydrogen bonded methanol and methoxonium ion (Figure 3a). Methoxonium ion is formed spontaneously when a methanol molecule captures the proton from the Brønsted acid site. No barrier was found for that reaction in agreement with the work of Sandre et al [8]. Clearly, the presence of the second methanol molecule in the zeolite cage facilitates the formation of a methoxonium ion formation. No spontaneous de-protonation of the zeolite was observed with one methanol molecule.

Figure 4 places the above two scenarios on the same footing. Note the pronounced differences in bond-length and angle between (a) one methanol molecule and (b) two methanol molecules, respectively.

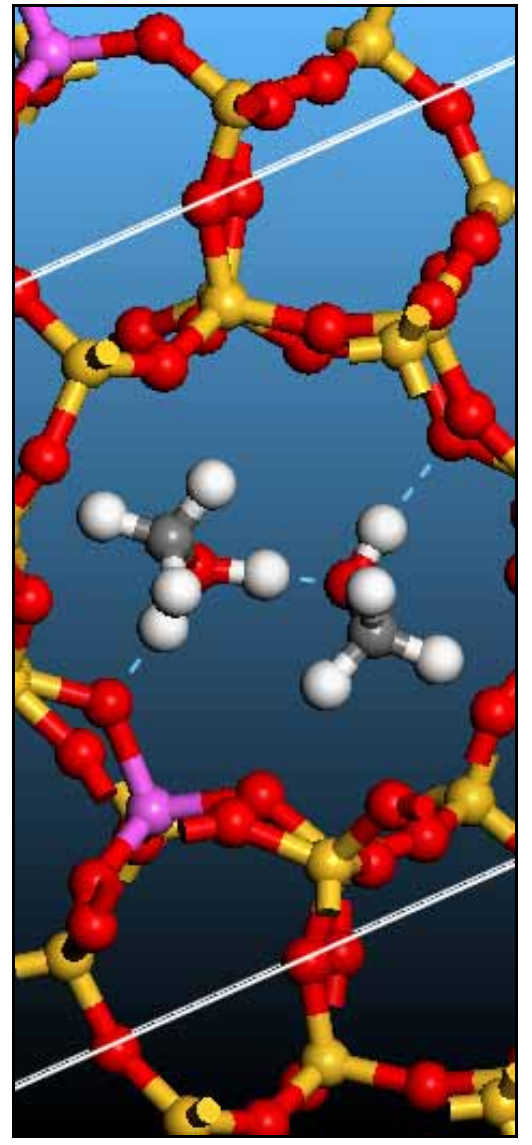

(a)

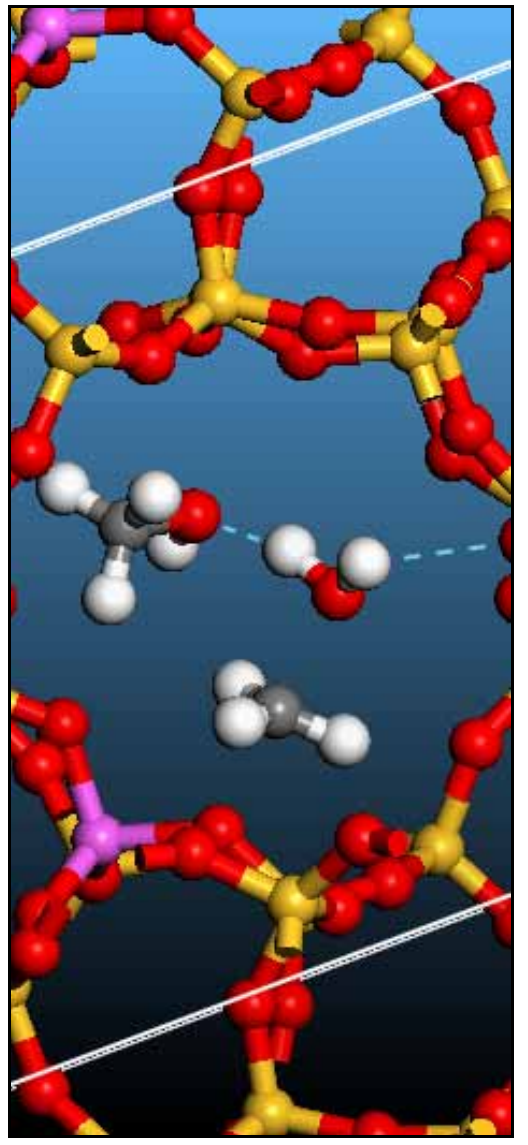

(b)

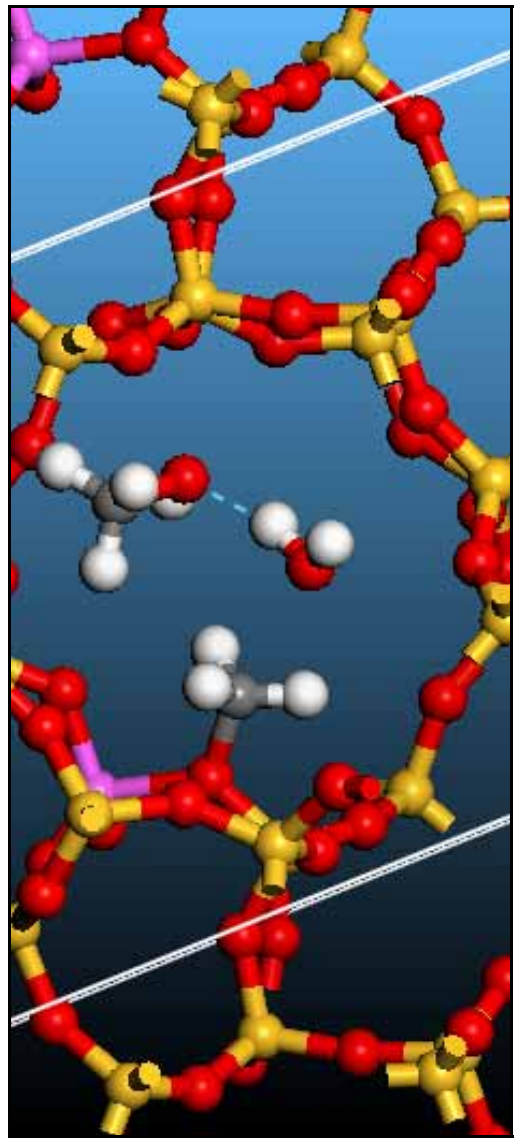

(c)

Figure 3. Surface methoxyl formation with two methanol molecules via a collinear $\mathrm{S}_{\mathrm{N}} 2$ type reaction.

(a) Hydrogen bonded methanol and methoxonium ion complex, (b) Transition state structure, (c) Surface methoxyl. Dotted lines represent hydrogen bonds. 


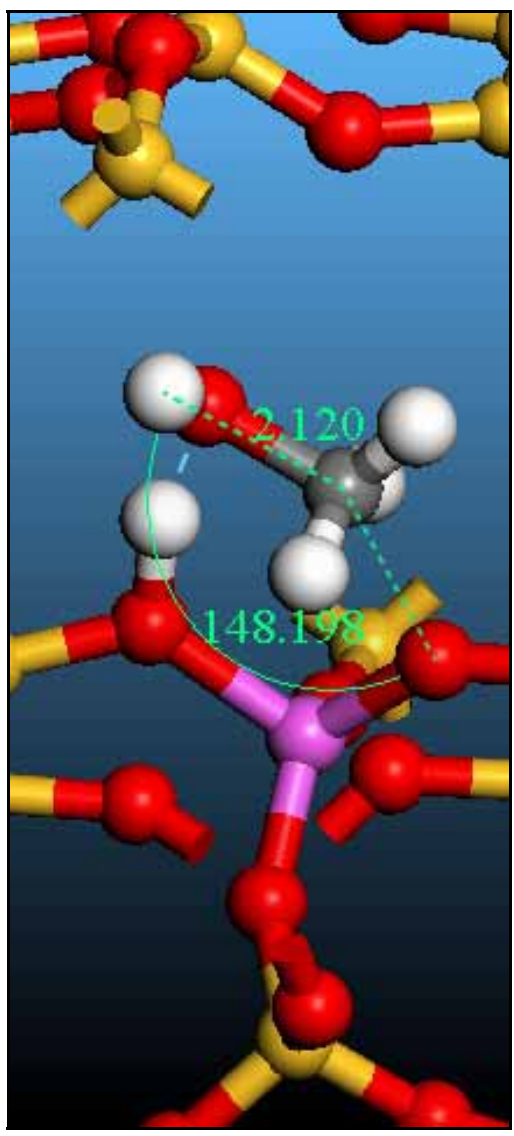

(a)

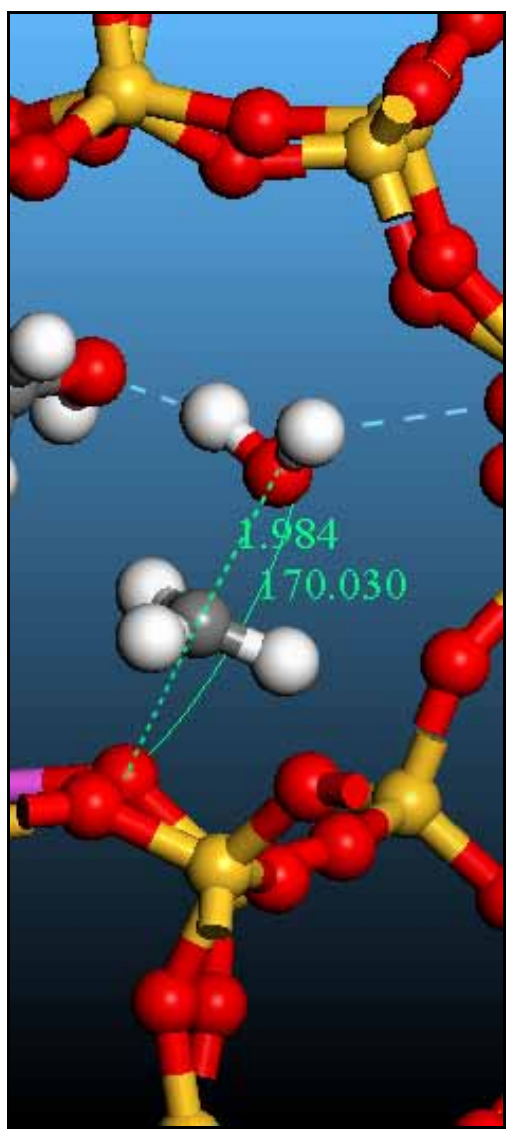

(b)

Figure 4. Transition state structures for surface methoxyl formation. (a) One methanol molecule, (b) Two methanol molecules. Dotted lines represent hydrogen bonds.

The surface methoxyl species can react with methanol or DME to form ethanol or methyl-ethylether, respectively [13]. These are the first species containing a C-C bond. In this study we have further investigated the formation of ethanol and have identified a different pathway than previously reported [1,13]. This pathway does not involve water as a catalyzing agent, the water molecule just acts as a spectator, playing no visible role in the $\mathrm{C}-\mathrm{C}$ bond formation (see Figure 5a). The transition state (Figure $5 b$ ) indicates that this is also a $\mathrm{S}_{\mathrm{N}} 2$ type reaction involving the $\mathrm{CH}_{3}$ species. However, this pathway involves a concerted reaction where the $\mathrm{CH}_{3} \mathrm{OH}$ fragment gives up a proton to the Brønsted acid site while simultaneously bonding with the methyl group. Transition state search calculations of this process yielded a barrier of $\sim 50 \mathrm{kcal} / \mathrm{mol}$. The overall reaction in this case is also exothermic (22 $\mathrm{kcal} / \mathrm{mol}$ ). To our knowledge we have not found any previous discussion in the literature about the plausibility of this pathway and believe this to be a competitive candidate to the previously reported process.

We have studied in detail a recent hypothesis [9,28] that a surface ylide $\left(\mathrm{CH}_{2}\right)$ can provide a reactive $\mathrm{C}$ atom for the first $\mathrm{C}-\mathrm{C}$ bond formation. According to that hypothesis the methoxyl species loses a proton that is transferred to the neighboring Brønsted acid site. Our attempts to find a stable structure with a surface ylide separated from a proton were not successful. The proton always returns, 


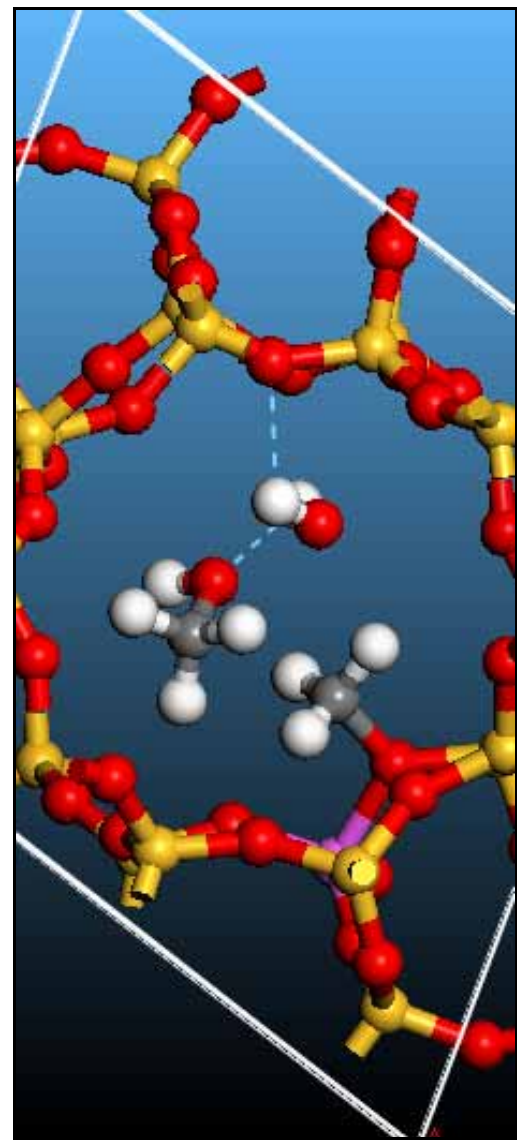

(a)

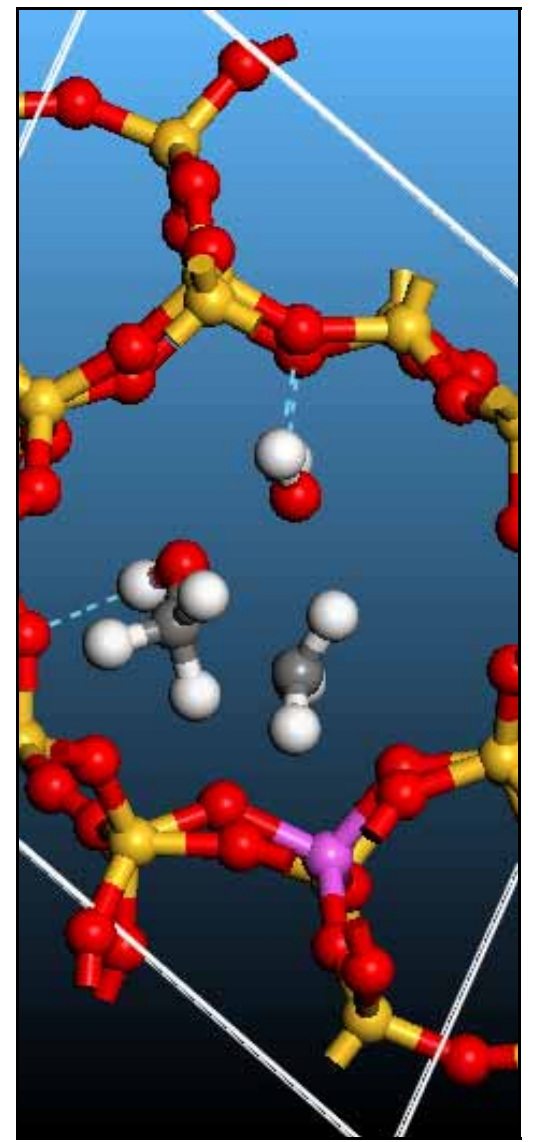

(b)

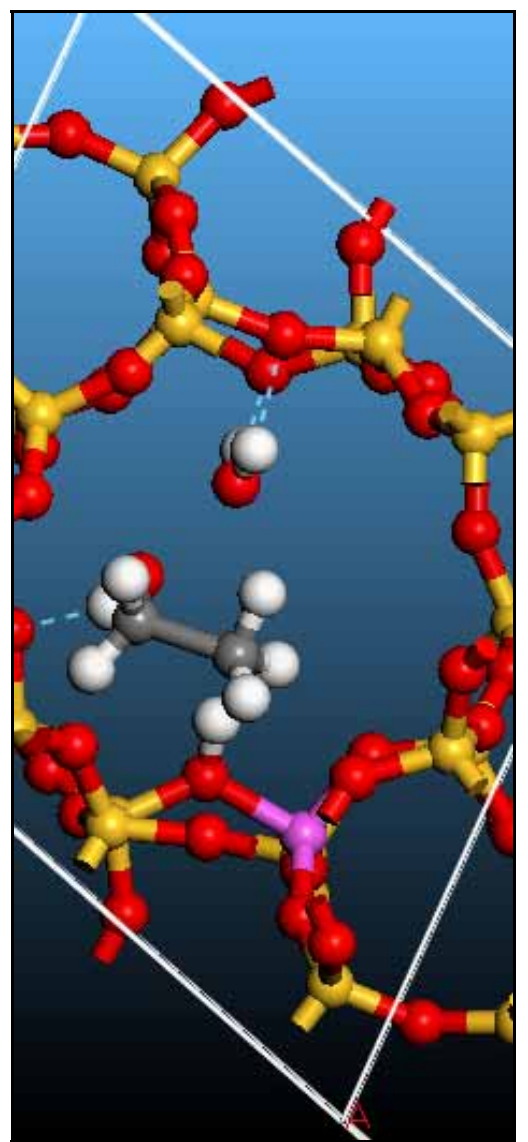

(c)

Figure 5. Formation of ethanol. Note that the water molecule is hydrogen bonded to the cage through the entire reaction and plays no visible role. This is a conventional $S_{N} 2$ type reaction involving the methoxyl species and the methanol molecule. (a) Surface methoxyl and methanol-water complex, (b) Transition state structure, (c) Ethanol molecule and proton transfer to Brønsted acid site. Dotted lines represent hydrogen bonds.

without any barrier to form a stable methoxyl species. However, with a rearrangement of the zeolite framework with the ylide $\left(\mathrm{CH}_{2}\right)$ species inserted into the Al-O bond, a stable intermediate structure is indeed possible. Cluster calculations $[9,28]$ reveal that the barrier for such reaction methoxyl $\Rightarrow$ ylide built into surface is about $50 \mathrm{kcal} / \mathrm{mol}$. We expect a small cluster model to be inadequate to study reactions that may significantly affect the entire framework of a zeolite. We investigated this reaction (Figure 6) using our periodic zeolite model. The calculated barrier of $78 \mathrm{kcal} / \mathrm{mol}$ is significantly higher compared with the other barriers for methoxyl $(44 \mathrm{kcal} / \mathrm{mol})$ and ethanol $(35 \mathrm{kcal} / \mathrm{mol}$ and 50 $\mathrm{kcal} / \mathrm{mol}$ ) formation, respectively. We, therefore, can rule out the possibility of ylide formation within the framework of our zeolite model. In Figure 7 we provide a detailed structure, along with representative bond lengths, around the Brønsted acid site. The structure clearly shows significant reorganization of the framework, underlining the need for calculations in a periodic environment. 


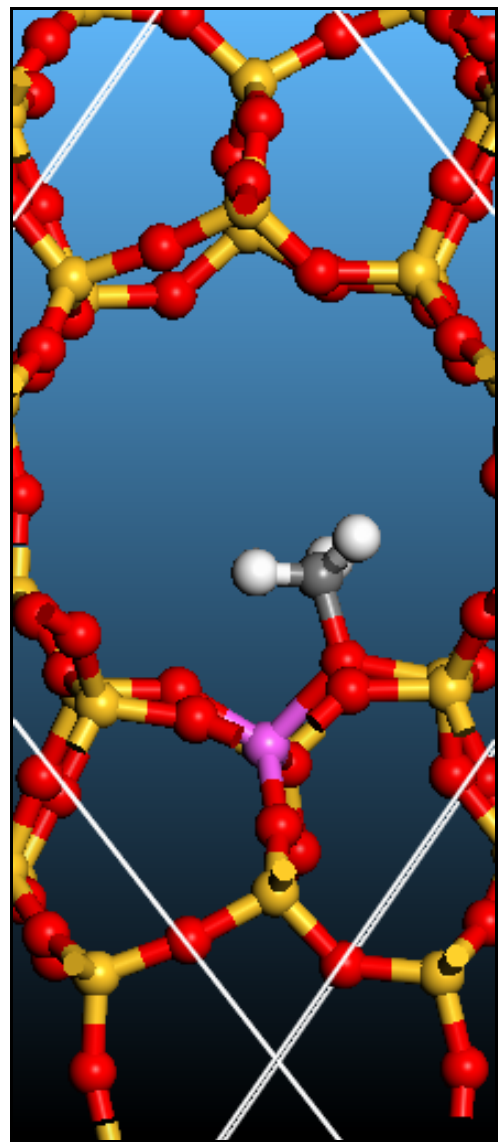

(a)

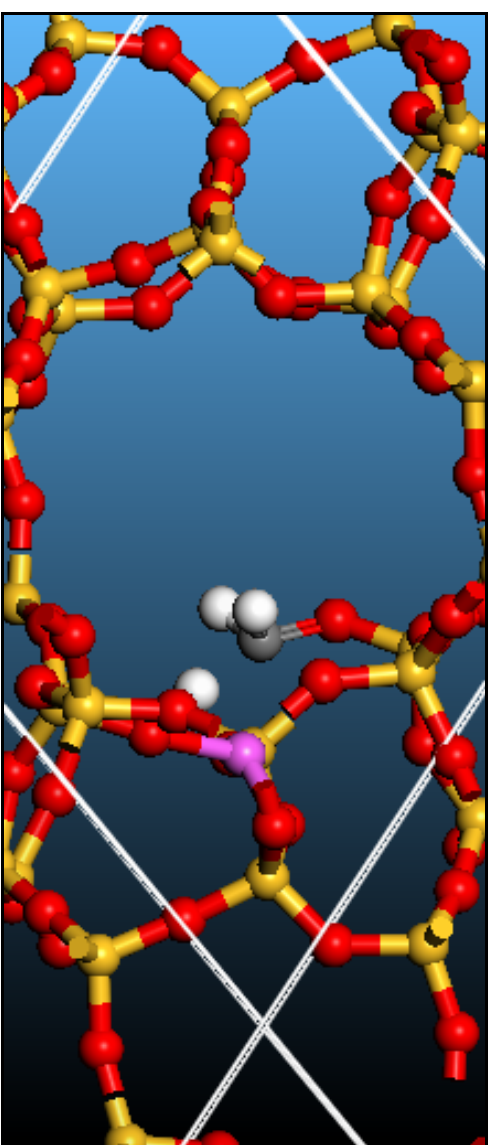

(b)

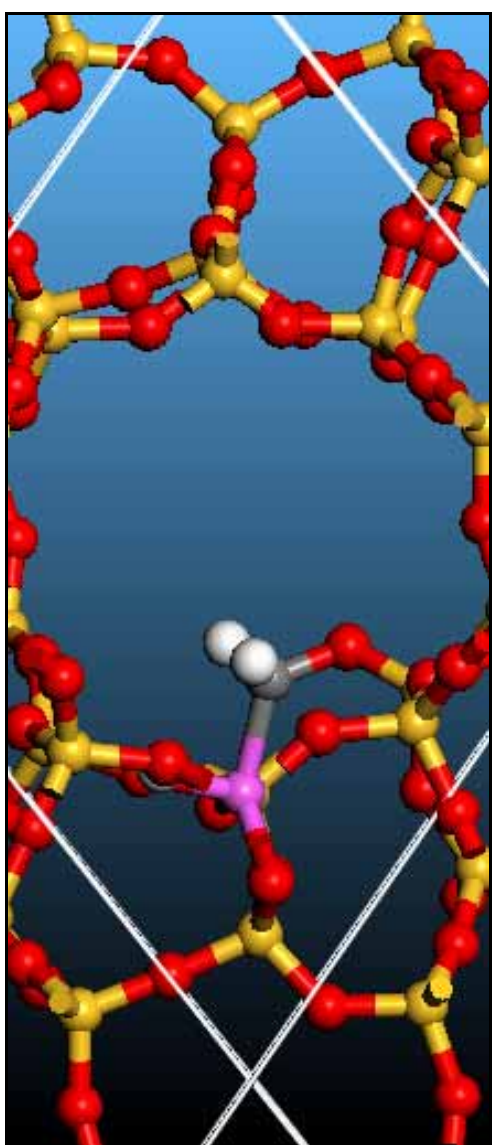

(c)

Figure 6. Incorporation of ylide into zeolite framework. (a) Surface methoxyl, (b) Transition state structure, (c) ylide structure incorporated into framework. Note the substantial lattice distortion around the Brønsted acid site as well as the zeolite cage.

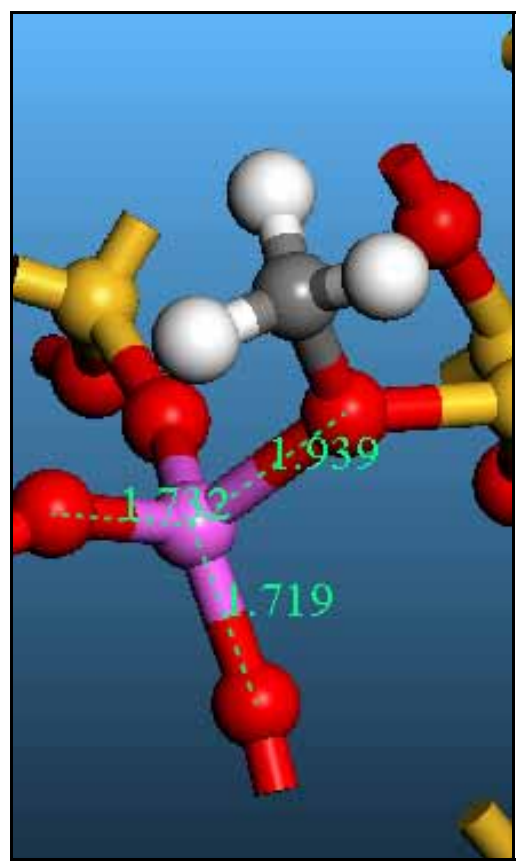

(a)

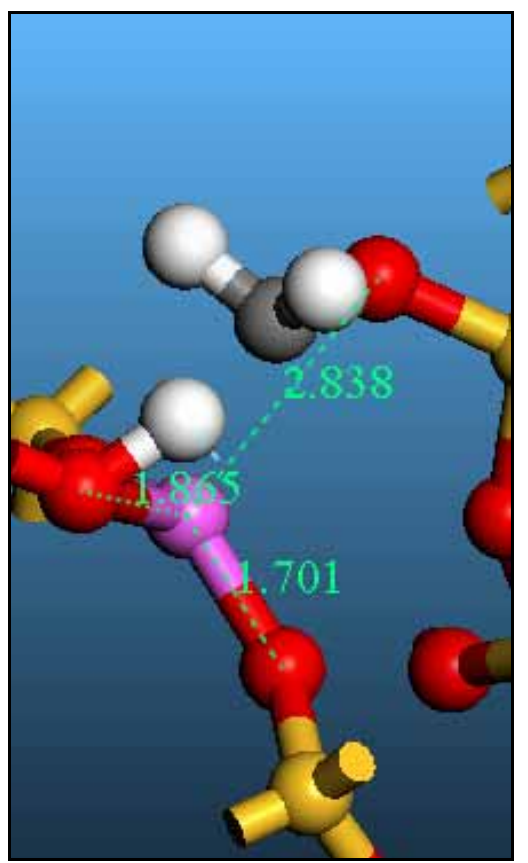

(b)

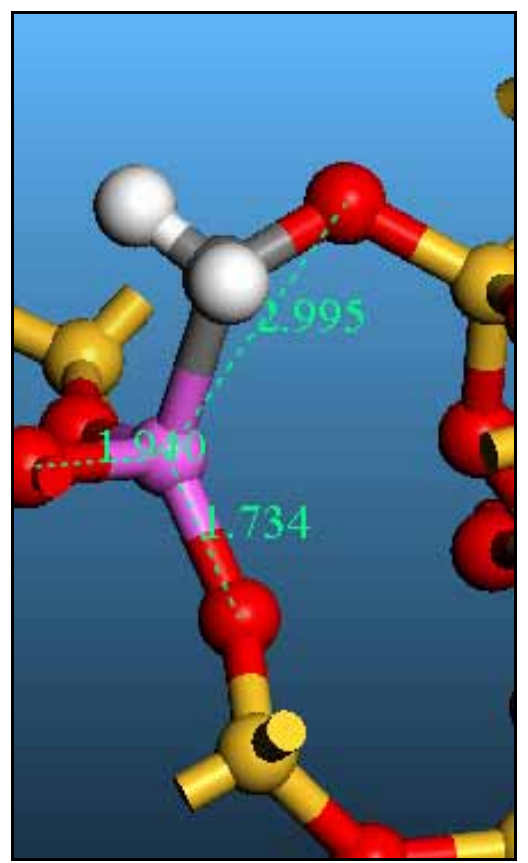

(c)

Figure 7. Local distortion around the Brønsted acid site. (a) Surface methoxyl, (b) Transition state structure, (c) ylide structure incorporated into the zeolite framework. 


\section{Conclusions}

We have performed all-electron DFT calculations on a periodic zeolite model (FER) using recently developed [18,19] geometry optimization and transition state search algorithms to elucidate the key reaction mechanisms involved in the breaking of the $\mathrm{C}-\mathrm{O}$ bond and the formation of the first $\mathrm{C}-\mathrm{C}$ bond in the MTG process. Periodic calculations are necessary to highlight the significant reorganization of the zeolite cage that is likely to occur, as we have shown above, if certain adsorbed species get built into the zeolite framework. We have shown that hydrogen bonds are described quite accurately $(\sim 0.5$ $\mathrm{kcal} / \mathrm{mol}$ ) within our approach. The neighborhood of the Brønsted acid site is also described well. The experimentally known positions of the bridging hydroxyl groups and their relative stabilities are correctly reproduced in our calculations on the alumino-silicate faujasite zeolite. Our calculations show that a single methanol molecule adsorbs to the zeolite cage via hydrogen bonds whereas two methanol molecules can lead to spontaneous formation of a methoxonium ion. The formation of surface methoxyl occurs via a $\mathrm{S}_{\mathrm{N}} 2$-type concerted reaction with a barrier of $44 \mathrm{kcal} / \mathrm{mol}$ if two methanol molecules are present. We have identified a new pathway for ethanol formation, a mechanism that does not require water as a catalyzing agent with a barrier of about $50 \mathrm{kcal} / \mathrm{mol}$. This is within the range of the barrier for the previously reported [1] mechanism (35 kcal/mol), indicating they could be competing schemes. The question of ylide formation was also addressed. Formation of surface ylide proved futile in disagreement with recent cluster calculations again emphasizing the importance of periodic calculations. Nevertheless, we were successful in stabilizing a ylide structure built into the zeolite. A very high reaction barrier of $78 \mathrm{kcal} / \mathrm{mol}$ was calculated for this process. This is not surprising because this reaction is accompanied by significant lattice distortion. One, therefore, can rule out the possibility of ylide formation within the framework of our periodic zeolite model. However, this still leaves an open question regarding the universality of this result which we believe can be answered with a systematic study using other models. We have not studied finite temperature effects in this work but expect that the overall picture should not be affected [27]. However, temperature effects could be relevant in determining the most stable of the weak van der Waal complexes and consequently influence the initial conditions. In this work the initial structures were carefully chosen after preliminary scans of the potential energy surface, so we expect them to be reasonable.

There are other issues that we have not addressed in this paper, i.e. the role of DME and the ethanol dehydration process to form ethylene. These are planned for a future project.

\section{Acknowledgement}

We are grateful to Prof. Richard Catlow (Royal Institute of Great Britain, London) and Prof. Joachim Sauer (Humboldt University, Berlin) for valuable discussions. We thank our colleagues at Accelrys for help in this study and acknowledge members of the Accelrys Catalysis 2000 Consortium for their support of this work. 


\section{References}

1. Andzelm, J.; Govind, N.; Fitzgerald, G.; Maiti, A. Int. J. Quantum Chem., submitted (2001).

2. Chang, C.D.; Silvestri, A.J. J. Catal. 1977, 47, 249.

3. Sauer, J. Chem. Rev. 1989, 89, 199.

4. Hutchings, G.J.; Hunter, R. Catal. Today 1990, 6, 279.

5. Haase, F.; Sauer, J. Micro. Meso. Mat. 2000, 35, 379.

6. Sinclair, P.E.; Catlow, C.R.A. J. Chem. Soc. Faraday Trans. 1996, 92, 2099.

7. Shah, R.; Gale, J.D.; Payne, M.C. J. Phys. Chem. 1996, 100, 11688.

8. Sandre, E.; Payne, M.C.; Gale, J.D. Chem. Commun. 1998, 22, 2445.

9. Hutchings, G.J.; Watson, G.W.; Willock, D.J. Micro. Meso. Mat. 1999, 29, 67.

10. Blaszkowski, S.R.; van Santen, R.A. J. Phys. Chem. 1995, 99, 11728.

11. Zicovich-Wilson, C.M.; Viruela, P.; Corma, A. J. Phys. Chem. 1995, 99, 13224.

12. Sinclair, P.E.; Catlow, C.R.A. J. Chem. Soc. Faraday Trans. 1997, 93, 333.

13. Blaszkowski, S.R.; van Santen, R.A. J. Am. Chem. Soc. 1997, 119, 5020.

14. Delley, B. J.Chem.Phys. 1990, 92, 508; J. Phys. Chem. 1996, 100, 6107; J. Chem. Phys. 2000, 113, 7756.

15. $\mathrm{DMol}^{3}$, Materials Studio 2.0, Accelrys Inc., San Diego, CA, USA.

16. Milman, V.; Winkler, B.; White, J.A.; Pickard, C.J.; Payne, M.C.; Akhmatskaya, E.V.; Nobes, R.H. Int. J. Quant. Chem. 2000, 77, 895.

17. CASTEP, Materials Studio 2.0, Accelrys Inc., San Diego, CA, USA

18. Andzelm, J.; King-Smith, D.; Fitzgerald, G. Chem. Phys. Lett. 2001, 335, 321; and to be published.

19. Govind, N.; Fitzgerald, G.; King-Smith, D. to be published

20. Perdew, J. P.; Burke, K.; Ernzerhof, M. Phys. Rev. Lett. 1996, 77, 3865.

21. Monkhorst, H. J.; Pack, J. D. Phys. Rev. B 1976, 13, 5188.

22. Szalewicz, K.; Jeziorski, B. In Molecular Interactions; Scheiner, S., Ed.; John Wiley \& Sons: New York, 1997; p. 3.

23. Klamt, A.; Jonas, V.; Burger, T.; Lohrenz, J. J. Phys. Chem. 1998, 102, 5074.

24. Kristian, S.; Pulay, P. Chem. Phys. Lett. 1994, 175, 229.

25. Czjzek, M.; Jobic, H.; Fitch, A.; Vogt, T. J. Phys. Chem. 1992, 96, 1535.

26. Hill, J-R.; Freeman, C.; Delley, B. J. Phys. Chem. A 1999, 103, 3772.

27. Haase, F.; Sauer, J. J. Am. Chem. Soc. 1995, 117, 3780.

28. Sinclair, P.E.; Catlow, C.R.A. J. Phys. Chem. 1997, 101, 295.

(C) 2002 by MDPI (http://www.mdpi.org), Basel, Switzerland. 